\title{
Preface
}

\section{Alzheimer's disease: A century of scientific and clinical research}

\author{
George Perry, Jesús Avila, June Kinoshita and Mark A. Smith
}

The centennial of Alois Alzheimer's original description of the disease that would come to bear his name offers a vantage point from which to commemorate the seminal discoveries in the field. To identify the breakthroughs, we used citation analysis, milestone papers identified by current researchers, and our own suggestions. Our process took into account the perspectives of individuals who recall the impact of findings at the time they were made, as well as of scientists today who have the advantage of hindsight in weighing the lasting influence of these findings. Because modern Alzheimer disease research was triggered by the seminal work of Tomlinson, Blessed, and Roth some four decades ago, we are especially fortunate that the vast majority of these milestone authors are still with us.

Each contributor was invited to discuss what made their particular article a milestone in the context of its time. Furthermore, contributors were asked to provide a highly personal perspective, by recounting the tale of how each discovery unfolded and by frankly describing the contradictions among studies and the debates that once took place in whispered tones in remote corners of seminar rooms and conference halls.

These writings bring to the practitioner, student and interested lay person a perspective not only on the past but also on where the Alzheimer disease field is likely to go in the future. Only time will tell whether these milestones have charted the future accurately, but they are unquestionably the foundation upon which the future will be built.

N.B. The photo's displayed on the title page of the papers show the (corresponding) author. 